\title{
Relative Importance of Different Attributes of Graphic Health Warnings on Tobacco Packages in Viet Nam
}

\author{
Kim Bao Giang ${ }^{*}$, Le Hong Chung², Hoang Van Minh², Vu Duy Kien², Vu Van \\ Giap$^{1}$, Nguyen Duc Hinh ${ }^{1}$, Nguyen Manh Cuong ${ }^{3}$, Pham Duc Manh ${ }^{3}$, Ha Anh \\ Duc $^{3}$, Jui-Chen Yang ${ }^{4}$
}

\begin{abstract}
Graphic health warnings (GHW) on tobacco packages have proven to be effective in increasing quit attempts among smokers and reducing initial smoking among adolescents. This research aimed to examine the relative importance of different attributes of graphic health warnings on tobacco packages in Viet Nam. A discrete choice experimental (DCE) design was applied with a conditional logit model. In addition, a ranking method was used to list from the least to the most dreadful GHW labels. With the results from DCE model, graphic type was shown to be the most important attribute, followed by cost and coverage area of GHW. The least important attribute was position of the GHW. Among 5 graphic types (internal lung cancer image, external damaged teeth, abstract image, human suffering image and text), the image of lung cancer was found to have the strongest influence on both smokers and non-smokers. With ranking method, the image of throat cancer and heart diseases were considered the most dreadful images. GHWs should be designed with these attributes in mind, to maximise influence on purchase among both smokers and non-smokers.
\end{abstract}

Keywords: Graphic health warnings - characteristics - relative importance - discrete choice experiment - Vietnam

Asian Pac J Cancer Prev, 17 Tobacco Prevention and Control in Vietnam Suppl, 79-84

\section{Introduction}

Graphic health warning (GHW) on tobacco package have been introduced and initially implemented in Canada since 2001 with setting coverage of $50 \%$ of the display area front and back. Up to date, 77 countries/jurisdictions have adopted the GHW label intervention and 60 countries/ jurisdictions have required coverage area of at least $50 \%$ of the tobacco package (Canadian Cancer Society, September, 2014). Many researches have been conducted to examine the effects of GHW label intervention on the tobacco control attempt and process.

GHWs have been reported as important channels for communicating tobacco harms to both smokers and nonsmokers, increasing the intention to quit smoking among users and preventing initial smoking (Thrasher et al., 2010; Hammond, 2011a; Durkin et al., 2015; Kotnowski et al., 2015; Li et al., 2015; Mead et al., 2015; Noar et al., 2015). It is estimated that each pack-per-day smoker is potentially exposed to GHW and tobacco package at least 7000 times per year during their smoking activities (Slade, 1997; Wakefield et al., 2002).

At the same time, non-smokers also have a high chance to encounter with pictorial warning messages on tobacco package as their public appearance are not only made by smokers but also by retailers (Hammond, 2011a). However, most studies focus on examining the impacts of specific graphic health warnings in specific contexts such as intention to quit, quitting attempts, preventing initial smoking, or deterring to buy tobacco as a gift; but very few studies evaluate the effect of different GHW designs and tobacco package on people's preference and choice to purchase (Kotnowski et al., 2015; Noar et al., 2015).

The first international treaty WHO Framework Convention on Tobacco Control (FCTC), established in 2003, provided guidance for implementing cigarette health warnings on tobacco package under Article 11, mainly to recommend rotating the health warnings that covered at least $30 \%$ of front and back display area of tobacco package and include images (World Health Organization, 2003). The six components of Tobacco control strategies - MPOWER, also stated "Health warnings on tobacco package reach all and cost governments nothing... Pictures of diseases have a greater impact than words alone"(World Health Organization, 2008).

In Vietnam, after great attempts, the Law on Prevention and Control of Tobacco Harms was passed in June 2012 and has been effective since May 1,2013. This is the first 
and comprehensive national control law related to tobacco harms prevention in Vietnam. Regarding packaging and labeling, the law required all cigarette packs to be printed out with 6 GHWs and covered $50 \%$ of front and back area of tobacco package. The GHWs are supposed to be revised and changed after every two years. To prepare for the GHW design revision and advocate for stronger tobacco prevention and control policies in Vietnam, this research examined the relative importance of different attributes of graphic health warnings on tobacco packages in Viet Nam.

\section{Materials and Methods}

To estimate the relative importance of attributes of graphic health warnings, we applied the discrete choice experiment (DCE) approach. DCE is a quantitative technique to elicit personal preferences for attributes of a product, a service or an intervention. The product (or intervention) of interest is illustrated by a series of attributes with varying levels. The selection of attributes and levels should meet three criteria: (1) the range of levels should be equivalent to what has been seen or might be seen in the market place, (2) differences in levels within each attributes should match what could be seen in the marketplace, and (3) the range of levels should include the maximum range over which target populations are willing to accept trade-offs among attributes. DCE has long been applied in market research to assist new product designs. DCE has also been widely used in environmental economics and health economics as well as many tobacco researches (Kotnowski et al., 2015). A typical DCE study allows researchers to quantify how much individuals value the attributes of a product (or an intervention in our proposed study) and to estimate marginal values (or willingness to pay [WTP]) of attributes.

We designed the DCE survey through a multi-step process. The process includes (a) identifying attributes and levels through literature review, scoping field visits, and cognitive interviews, (b) drafting DCE questionnaire for pretesting, (c) developing the experimental design of choice sets in SAS, which evaluates the hypothetical warning label formats, (d) finalizing the DCE questionnaire.

In the DCE survey design, we finally constructed 9 blocks and in each block a series of 8 choice questions and one repeated choice question was applied, based on experimental design in SAS. We assessed the impacts of the 4 actual GHW designs that have been used in Viet Nam and other potential important attributes of cigarette packs. Below a list of draft attributes and levels was presented. The actual attributes and levels were determined and finalized based on field tests, and pretesting of draft survey instrument (Table 1).

To predict potential changes in the effects of GHW designs, we conducted two rounds of DCE exercise in June 2014 and January 2015. In each survey round, 2634 respondents aged 15 and above were interviewed. The sample size above was calculated based on the guide by Johnson et al. (2013). Firstly, three region-based stratifications were identified (the North, the Center and the South). In each selected region, we classified communes (primary sampling units-PSUs) into 4 groups regarding living area (urban and rural area) and economic situation (poor and non-poor communes).

All data were analysed using Stata software version 12. The DCE data were analyzed using a conditional logit model. Responses to the choice questions (i.e., purchasing cigarette Pack A, or purchasing cigarette pack B, or neither for smokers; and pack A deters purchase, or pack $\mathrm{B}$ deters purchase, or neither for non-smokers) were the dependent variables. The levels of each attribute listed in Table 1 were the independent, categorical variables that were effects-coded. With effects coding, zero indicates the mean effect across all attribute levels, rather than the omitted attribute level as in dummy coding (Hensher et al., 2005). This procedure produces a coefficient for all attribute levels, where the coefficient for the omitted attribute level is the negative sum of those for the

Table 1. Attributes and Levels of the DCE Questions

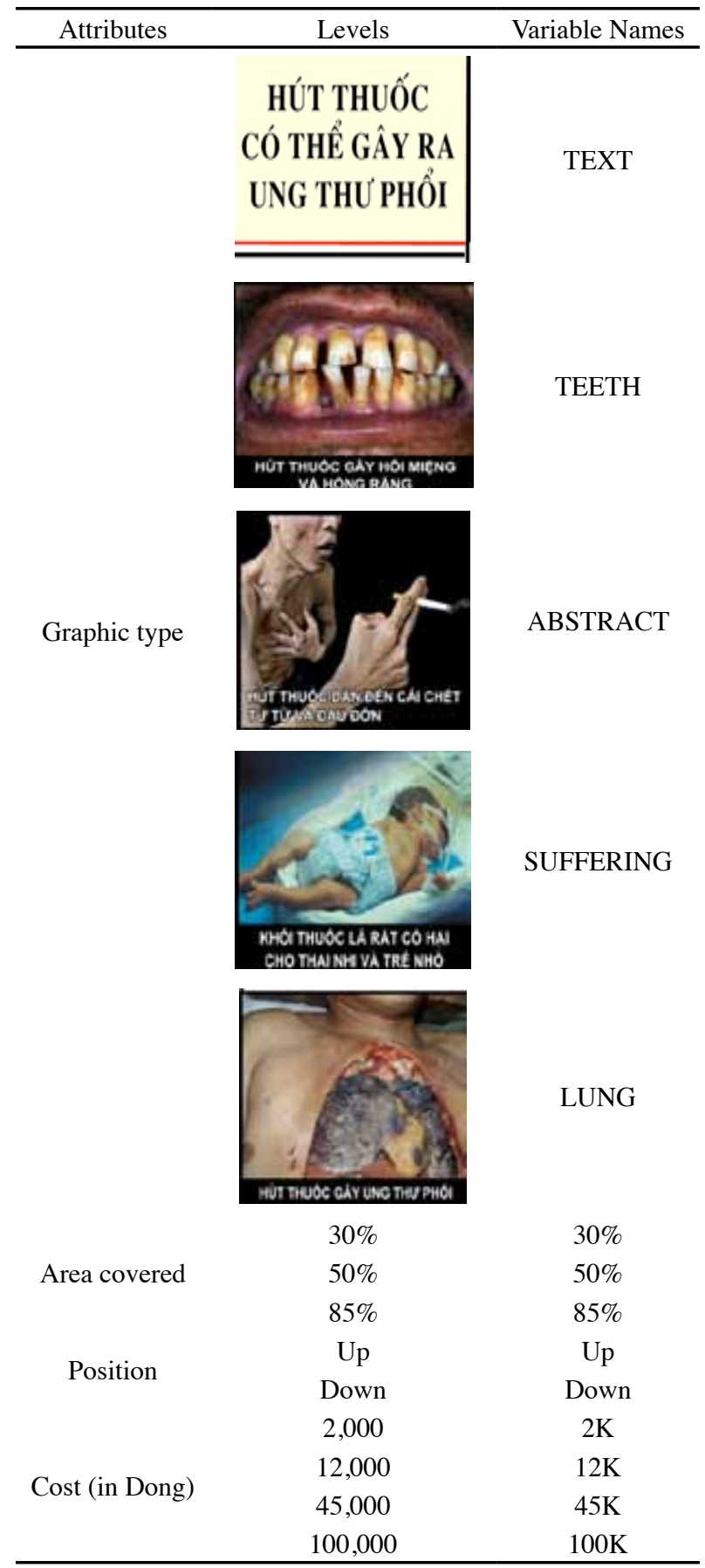


included attribute levels. The resulting coefficients can be interpreted as preference weights, where t-statistic values indicate whether preference weights differ significantly from zero (i.e., the mean effect), not from an omitted attribute level. Also, middle attribute levels often are close to the mean effect, and thus will have low significance. If the confidence intervals (calculated at the 95\% level) around any two preference weights did not overlap, the differences between the preference weights would be statistically significant at the $5 \%$ level or better $(\mathrm{P} \leq 0.05)$.

\section{The following conditional logit model was estimated}

$$
\begin{aligned}
& \mathrm{U}=\beta_{\mathrm{TEXT}} \times \mathrm{TEXT}+\beta_{\mathrm{TEETH}} \times \mathrm{TEETH}+\beta_{\mathrm{ABSTRACT}} \times \mathrm{ABSTRACT}+ \\
& \beta \pm \times \mathrm{SUFFER}+\beta \pm \times \mathrm{LUNG}+ \\
& \beta_{30 \%} \times 30 \%+\beta_{50 \%} \times 50 \%+\beta_{85 \%} \times 85 \%+ \\
& \beta_{\mathrm{UP}} \times \mathrm{UP}+\beta_{\mathrm{DOWN}} \times \mathrm{DOWN}+ \\
& \beta_{2 \mathrm{~K}} \times 2 \mathrm{~K}+\beta_{12 \mathrm{~K}} \times 12 \mathrm{~K}+\beta_{45 \mathrm{~K}} \times 45 \mathrm{~K}+\beta_{100 \mathrm{~K}} \times 100 \mathrm{~K},
\end{aligned}
$$

where $U$ is the implicit utility for a particular cigarette package. In the case of smokers, $\boldsymbol{\beta}_{\mathrm{TEXT}}, \boldsymbol{\beta}_{\mathrm{TEETH}}, \boldsymbol{\beta}_{\mathrm{ABSTRACT}}$, $\beta_{\text {SUFFER }}, \beta_{30 \%}, \beta_{50 \%}, \beta_{\mathrm{UP},} \beta_{2 \mathrm{~K}}, \beta_{12 \mathrm{~K}}$ and $\beta_{45 \mathrm{~K}}$ were explicitly estimated in the model; $\beta_{\text {LUNG }}$ was calculated as the negative sum of $\beta_{\text {TEXT }}, \beta_{\text {TEETH }}, \beta_{\text {ABSTRACT }}$, and $\beta_{\text {SUFFER }} ; \beta_{85 \%}$ was the negative sum of $\beta_{30 \%}$ and $\beta_{50 \%} ; \beta_{\text {Down }}$ equalled $\beta_{\mathrm{UP}}$ times minus one; and $\beta_{100 \mathrm{~K}}$ was the negative sum of $\beta_{2 \mathrm{~K}}, \beta_{12 \mathrm{~K}}$, and $\beta_{45 \mathrm{~K}}$.

In the case of non-smokers, $\beta_{\text {TEETH }}, \beta_{\text {ABSTRACT }}, \beta_{\text {SUFFER }}$, $\beta_{\text {LUNG }}, \beta_{50 \%}, \beta_{85 \%}, \beta_{\text {DOWN }}, \beta_{12 \mathrm{~K}}, \beta_{45 \mathrm{~K}}, \beta_{100 \mathrm{~K}}$ were explicitly estimated in the model; $\beta$ TEXT was calculated as the negative sum of $\beta_{\text {TEETH }}, \beta_{\text {ABSTRACT }}, \beta_{\text {SUFFER }}$, and $\beta_{\text {LUNG }} ; \beta_{30 \%}$ was the negative sum of $\beta_{50 \%}$ and $\beta_{85 \%} ; \beta$ UP equalled $\beta_{\text {Down }}$ times minus one; and $\beta_{2 \mathrm{~K}}$ was the negative sum of $\beta_{12 K}, \beta_{45 K}$, and $\beta_{100 K}$. To account for the panel nature of the data (i.e., multiple observations from each survey respondent), the model clustered standard errors at the survey respondent and choice question levels.

Preference weights for different attributes of cigarette packs can be interpreted in the form of relative importance (Train, 2003; Hensher et al., 2005). There are 2 types of relative importance:

Unscaled relative importance: Within each attribute, the vertical distance between any two preference weights is called the relative importance. The greater the vertical distance, the greater the importance placed on that change in the attribute levels. The distance between preference weights for the best level and the worst level of an attribute can be interpreted as the overall relative importance of the attribute over the range of levels assessed in the survey. The relative importance of changes within one attribute can also be compared with the relative importance of changes within other attributes to examine the trade-offs that people would be willing to make across attributes.

Scaled relative importance: The most important attribute was assigned an importance weight of 10. All the other attributes were scaled relative to the most important attribute.The DCE data were effects-coded and analyzed using a conditional logit model. The resulting coefficients can be interpreted as preference weights. If the confidence intervals (calculated at the 95\% level) around any two preference weights did not overlap, the differences between the preference weights would be statistically significant at the $5 \%$ level or better $(\mathrm{P} \leq 0.05)$ (Hensher et al., 2005). Within each attribute, the vertical distance between any two preference weights is called the relative importance. The greater the vertical distance, the greater the importance placed on that change in the attribute levels. In addition, the relative importance of changes within one attribute can be compared with the relative importance of changes within a different attribute to examine the trade-offs that smokers and non-smokers would be willing to make across attributes. The overall relative importance of each attribute can be calculated by taking the difference between the largest and smallest preference weights of the given attribute.

\section{Results}

Figure 1 shows the preference weights (or coefficients) of attributes levels from the conditional logit model for smokers. A positive sign on a preference weight (or coefficient) indicates an increase in utility of smokers while a negative indicates a decrease in utility. The results from the two rounds of our research showed similar preferences among smokers. Among graphic types, the text option was the most preferred while the image of lung cancer was the least preferred. In terms of area covered, smokers preferred smaller GHW labels to larger ones on the cigarette packaging. The negative coefficient of "down" position illustrated that upper position was preferred. As expected, smokers preferred lower cost

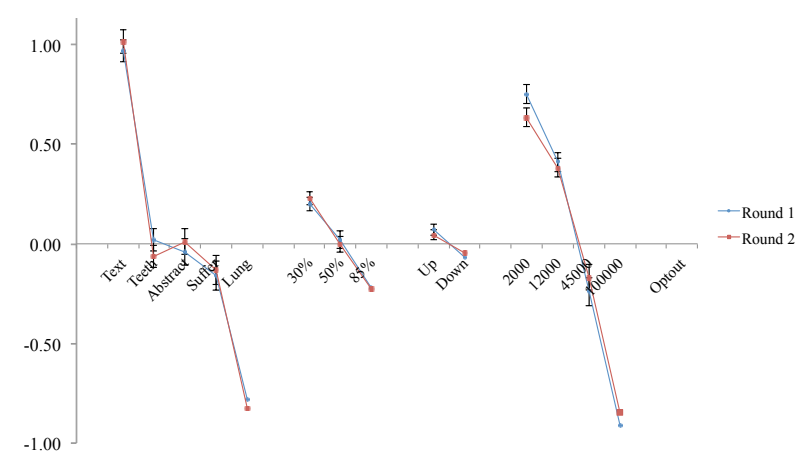

Figure 1. Smoker's Preference Weights for Different Attributes of Cigarette Packs

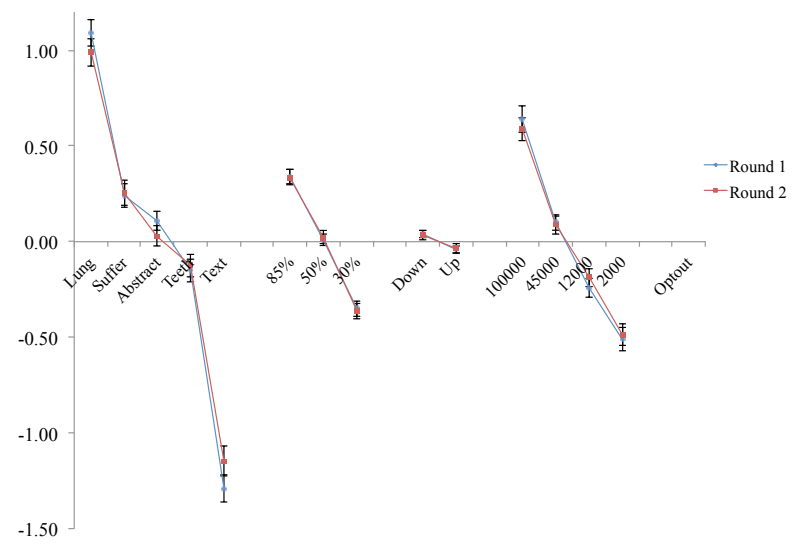

Figure 2. Nonsmoker's Preference Weights for Different Attributes of Cigarette Packs 


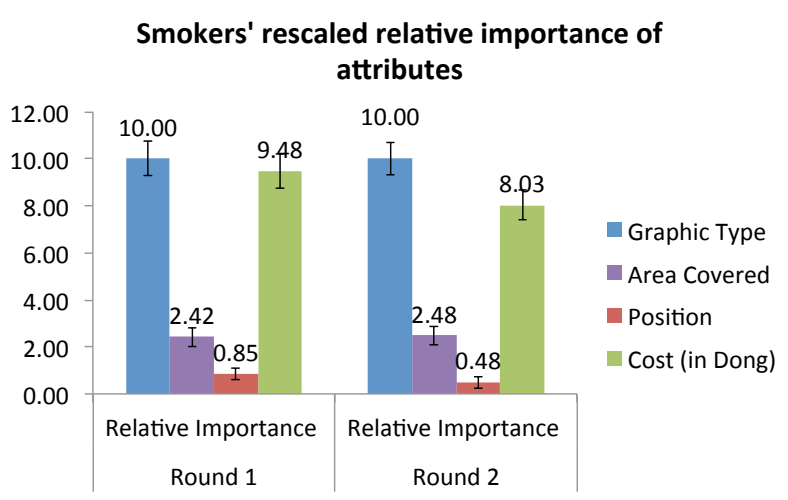

Figure 3. Rescaled Relative Importance of Different Attributes of Cigarette Pack Ranked by Smokers

Table 2. Ranking Order of 6 Graphic Health Warning Labels Among Smokers

\begin{tabular}{lcccc}
\hline Graphic types & \multicolumn{2}{c}{ Round 1 } & \multicolumn{2}{c}{ Round 2 } \\
\cline { 2 - 5 } & Mean & SD & Mean & SD \\
\hline Throat & 4.84 & 1.18 & 4.96 & 1.11 \\
Heart & 4.52 & 1.25 & 4.54 & 1.19 \\
Lung & 4.57 & 1.16 & 4.47 & 1.21 \\
Abstract & 2.57 & 1.21 & 2.42 & 1.20 \\
Suffering & 2.30 & 1.46 & 2.34 & 1.50 \\
Teeth & 2.20 & 1.27 & 2.27 & 1.24 \\
\hline
\end{tabular}

Table 3. Ranking Order of 6 Graphic Health Warning Labels among Non-Smokers

\begin{tabular}{lcccc}
\hline Graphic types & \multicolumn{2}{c}{ Round 1 } & \multicolumn{2}{c}{ Round 2 } \\
\cline { 2 - 5 } & Mean & SD & Mean & SD \\
\hline Throat & 4.92 & 1.16 & 4.98 & 1.12 \\
Heart & 4.54 & 1.24 & 4.48 & 1.30 \\
Lung & 4.55 & 1.15 & 4.47 & 1.18 \\
Abstract & 2.59 & 1.20 & 2.49 & 1.18 \\
Suffering & 2.34 & 1.39 & 2.48 & 1.48 \\
Teeth & 2.05 & 1.21 & 2.09 & 1.20 \\
\hline
\end{tabular}

to higher cost. To be specific, they preferred a tobacco package that costs $2,000 \mathrm{VND}$ or $12,000 \mathrm{VND}$ to one that costs 45,000 VND or more.

Figure 2 presents the preference weights of attribute levels from the conditional logit model for non-smokers. As opposed to smokers, non-smokers preferred the image of the lung cancer most and the text option least. In terms of area covered, non-smokers preferred larger GHW labels to smaller ones on the cigarette packaging. The negative coefficient of "up" position illustrated that position "down" was preferred. As expected, non-smokers preferred higher cost to lower cost. To be specific, they preferred a tobacco package that costs over 45,000 VND to the cheaper ones.

Given the range of attributes and levels in the study, the attribute "Graphic type" was the most important attribute to smokers in both Round 1 and Round 2, which was assigned an importance weight of 10. All the other attributes were scaled relative to the most important attribute. In Round 1, the attributes were ranked as follows: Graphic type (10), Cost (9.5), Area covered (2.4), and Position (0.8). Using the same logic, in the Round 2, the attributes were ranked as follows: Graphic type

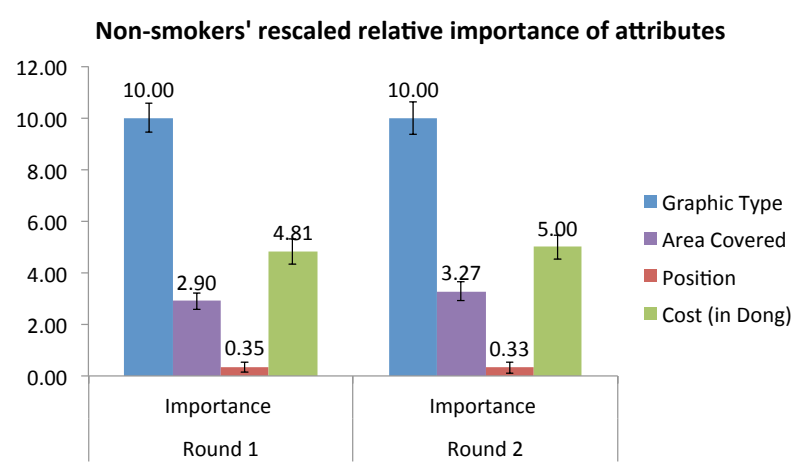

Figure 4. Scaled Relative Importance of Different Attributes of Cigarette Pack Ranked by Non-Smokers

(10), Cost (8.0), Area covered (2.5), and Position (0.5). In another word, Graphic Type was at least 4 times as important as Area Covered and Graphic Type was more than 10 times as important as Position. Graphic Type was as important as Cost. Area Covered was at least twice as important as Position.

Like smokers, the attribute "Graphic type" was the most important attribute to non-smokers in Round 1 and Round 2, which was assigned an importance weight of 10 . All the other attributes were scaled relative to the most important attribute. In Round 1, the attributes were ranked as follows: Graphic type (10), Cost (4.8), Area covered (2.9), and Position (0.4). In Round 2, the attributes were ranked as follows: Graphic type (10.0), Cost (5.0), Area covered (3.3), and Position (0.3). In another word, Graphic Type was twice as important as Cost. Graphic Type was at least 3 times as important as Area Covered. Graphic Type was least 30 times as important as Position. Area Covered was at least 8 times as important as Position (Figure 4).

In order to impute relative importance for the two GHW labels (the image of throat cancer and the image of heart diseases) that were not part of the DCE design, we asked respondents to rank 6 GHW labels of interest to this study, 1 being the most dreadful and 6 being the least dreadful. For analytical purposes, the most dreadful label was assigned a value of 6 , and the least dreadful label was assigned a value of 1 . A higher mean value meant a higher rank order.

Among smokers, throat cancer was ranked the highest in both phases. Heart diseases and lung cancer ranked third and second in Round 1 and second and third in Round 2, respectively. Teeth image was seen as the least scary image in our study (Table 2). In general, the ranking results were consistent with the results from the DCE model, except for the case of human suffering image and abstract. This may be explained by the facts that the people are also concerned of other factors when trading off on attributes such as the relevance to their children's health, the severity and reality of the image rather the fearness of the image. Non-smokers showed similar preferences. The images of throat cancer, heart diseases and lung cancer were the most frightening to non-smokers (Table 3).

\section{Discussion}

This study was the first in Vietnam and among the 
earliest ones in the world to examine the impact of implementing GHW labels on users' preference and choice using DCE approach. Previous studies on graphic health warnings focused on assessing their impacts on a consumer's attention to warnings, cognitive and emotional reactions to warnings, and change in beliefs about the harmful effects of smoking (Noar et al., 2015). Our study results provide valuable information on how smokers and non-smokers weighted, evaluated and decided on each GHW labels and other attributes of a tobacco package directly. According to our estimations, the GHW labels and price of a tobacco package were the two attributes with the greatest influence on the participants' choice. These findings can provide the first tangible targets for intervention and regulatory strategies on tobacco control and prevention.

Among 4 attributes and across levels evaluated, the Graphic Type, especially with the image of lung cancer, had the strongest influence on both smokers and nonsmokers' preferences to not purchase tobacco. Next was the image of human suffering. Text-only was the least effective type of warning to curb smoking. This result is supported by previous research, reviews and from a meta-analysis, which claimed that GHW labels were more effective than text-only warning labels (Disease, 2009; Hammond, 2009; 2011b; Wardle et al., 2010; TNS Qual, 2012; Noar et al., 2015). The reason for smokers' preference on tobacco package without graphic health warnings can be explained by their defensive mechanism /reflexive response (Moodie C; Kessels LT, 2010). Other research has previously suggested that even smokers who have accepted tobacco's harmful consequences may still try to ignore graphic health warnings due to their addiction or as an indication of low motivation to quit smoking (TNS Qual, 2012). However, despite their refutation, graphic health warnings have proven their effectiveness in reducing smoking rates, encouraging smoking cessation among adult smokers and preventing smoking among adolescents (Hammond, 2006; Fong and Hitchman, 2009). According to the findings from a cohort study conducted by The International Tobacco Control Policy Evaluation Project and from other researchers, GHW labels had shown effectiveness in reducing cigarette consumption, encouraging smoking cessation and preventing new smokers (O'Hegarty et al., 2006; Hammond, 2008; Shanahan, 2009). In the case of the image of human suffering, many researchers suggested that health warning labels with pictures expressing the risk of secondhand smoke on babies and infants elicited emotional responses from smokers, especially those who have children (Elliott \& Shanahan Research, 2008). In an evaluation to test the new health warning labels conducted in European countries, the health warning labels with pictures related to potential harm for children were some of the most effective amongst smokers (TNS Qual, 2012).

Price was found to be the second most important attribute for influencing product preference. Overall, smokers were more likely to choose less expensive tobacco package, while non-smokers preferred the more expensive one. These findings were consistent with the law of demand. In addition, increasing tobacco price was proved as the most effective method to reduce smoking prevalence and tobacco demand. It also could prevent new smokers, especially younger individuals (Chaloupka, 2000). Price-based policy and taxation therefore may be feasible options for an effective smoking regulation framework.

The third attribute strongly associated with preferences was the area covered by health warning labels. On average, smokers preferred the smaller health warning labels while non-smokers were more likely to choose the bigger labels. Increasing the current covered area $(>50 \%)$ may help to increase the effectiveness of the intervention due to making GHW labels more obvious and deter purchase more effectively. This has been supported by previous research; however, Wakefield et al. (2012) showed that the disappearance of brand-name has been more effective in reducing tobacco appeal than increasing the size of health warning labels. Therefore, plain packaging should be included in additional research as a physical attribute of tobacco package.

The discrete choice experimental results show that the position of health warning labels was less important with smokers than other attributes. This result was the same as the result of one particular study conducted in New Zealand in 1987. The authors indicated that there was no difference between the lower and upper position of tobacco package. In 2008, WHO also required Parties to establish the health warnings on the uppermost position of the tobacco package.

In order to explore the relative importance for the two GHW labels (the image of throat cancer and the image of heart diseases) that were not part of the DCE design, we applied the ranking method, to arrange these 6 pictures from the most deterrent to least deterrent. The image of throat cancer became the most repulsive while the image of heart diseases was ranked in the second or third order, together with the lung cancer image. Since photos with more aversive qualities were previously shown to increase smoking cessation engagement (Noar et al., 2015), our results suggest that the other two labels may have had greater weight on both smokers and non-smokers' preference than the 4 images included in our DCE design. To elicit the important weights of current and future GHW labels, it is suggested that the DCE approach needs to be conducted regularly and actively.

Regarding limitations, we only evaluated the impact of 4 among 6 current GHWs available on the market. Conducting further research to examine the effects of GHWs labels in a wider setting and using advance methods could provide richer information and evidence for policy advocacy and implementation.

\section{Acknowledgements}

We thank International Development Research Centre (IDRC) for providing funding support for this study. We also would like to express our sincere appreciation to Dr. Malcolm Moore, the Editor-in-Chief of the Asian Pacific Journal of Cancer Prevention, for his significant input to this paper. We would like to thank Phuong Tran for editing the English of this paper. 


\section{References}

Chaloupka FJ (2000). The Economics of Smoking. Handbook Health Economics, 1, 1539-627.

Disease IUATaL (2009). Tobacco packaging and labelling: Technical Guide, 7.

Durkin S, Brennan E, Coomber K, et al (2015). Short-term changes in quitting-related cognitions and behaviours after the implementation of plain packaging with larger health warnings: findings from a national cohort study with Australian adult smokers. Tob Control, 24, 26-32.

Elliott and Shanahan Research (2008). Literature review: evaluation of the effectiveness of the graphic health warnings on tobacco product packaging 2008. Australian Government Department of Health and Ageing, 34-5.

Fong GT, Hitchman SC (2009). The impact of pictures on the effectiveness of tobacco warnings. Bull WHO, 87, 640-3.

Hammond D (2008). Health warnings on tobacco packageages: Summary of evidence and legal challenges. Waterloo: University of Waterloo Report.

Hammond D (2011a). Health warning messages on tobacco products: a review. Tob Control, 20, 327-37.

Hammond D (2011b). Health warning messages on tobacco products: a review. Tob Control., 20, 327-37.

Hammond D FG, McNeill A, Borland R, Cummings KM (2006). Effectiveness of graphic health warnings in informing smokers about the risks of smoking: Findings from the International Tobacco Control (ITC) four country survey. Tob Control, 15, 19-25.

Hammond D (2009). Tobacco labelling \& packaging toolkit: a guide to FCTC Article 11. Tobacco Labelling Resource Centre.

Hensher DA, Rose JM, Greene WH (2005). Applied Choice Analysis. Cambridge: Cambridge University Press.

Kessels LT RR, Jansma BM (2010). Increased attention but more efficient disengagement: neuroscientific evidence for defensive processing of threatening health information. Health Psychol, 29, 346-54.

Kotnowski K, Fong GT, Gallopel-Morvan K, et al (2015). The impact of cigarette packaging design among young females in canada: findings from a discrete choice experiment. Nicotine Tob Res, ntv114. Epub 2015/05/28.

Li L, Borland R, Yong H, et al (2015). Longer term impact of cigarette package warnings in Australia compared with the United Kingdom and Canada. Health Educ Res, 30, 67-80.

Mead EL, Cohen JE, Kennedy CE, et al (2015). The role of theory-driven graphic warning labels in motivation to quit: a qualitative study on perceptions from low-income, urban smokers. BMC Public Health, 15, 92.

Moodie C MA, Hastings G (2013). Adolescents' response to pictorial warnings on the reverse panel of cigarette packs: A repeat cross-sectional study. Tob Control, 24, e93-e7.

Noar SM, Hall MG, Francis DB, et al (2015). Pictorial cigarette pack warnings: a meta-analysis of experimental studies. Tob Control, (Epub ahead of print).

O’Hegarty M, Nelson DE, Mowery P, Gable JM, Wortley P (2006). Reactions of young adult smokers to warning labels on cigarette package. Am J Prev Med, 30, 467-73.

Reed Johnson F, Lancsar E, Marshall D, et al (2013). Constructing experimental designs for discrete-choice experiments: report of the ISPOR conjoint analysis experimental design good research practices task force. Value Health, 16, 3-13.

Shanahan P (2009). Evaluation of the Effectiveness of Graphic Health Warnings on Tobacco Product Packaging 2008Executive Summary,. Australian Department of Health and Ageing.

Slade J (1997). The pack as advertisement. Tob Control, 6,
169-70.

Thrasher JF, Villalobos V, Szklo A, et al (2010). Assessing the impact of cigarette package health warning labels: a crosscountry comparison in Brazil, Uruguay and Mexico. Salud Publica Mex, 52, 206-15.

TNS Qual (2012). Tobacco packageaging health warning labels. Train K (2003). Discrete Choice Methods with Simulation. Cambridge: Cambridge University Press.

Wakefield M, Durkin S, Hammond D, Goldberg M, Borland R (2012). Do larger pictorial health warnings diminish the need for plain packaging of cigarettes? Addiction, 107, 1159-67.

Wakefield M, Morley C, Horan JK, et al (2002). The cigarette pack as image: new evidence from tobacco industry documents. Tob Control, 11, 73-80.

Wardle H, Lee L, Hall J, et al (2010). Evaluating the impact of picture health warnings on cigarette packets. Public health research consortium short report, Department of Health Policy Research programme, London, UK. 